\title{
INOVAÇÃO E DESEMPENHO ORGANIZACIONAL: UM ESTUDO DAS PUBLICAÇÕES CIENTÍFICAS DA BASE WEB OF KNOWLEDGE
}

\author{
INNOVATION AND ORGANIZATIONAL PERFORMANCE: A STUDY OF SCIENTIFIC \\ PUBLICATIONS OF THE WEB OF KNOWLEDGE BASE
}

Recebido em 18.02.2013. Aprovado em 01.10.2013

Avaliado pelo sistema double blind review

DOI: http://dx.doi.org/10.12712/rpca.v7i4.271

\begin{abstract}
Washington Romão dos Santos
washington_romao@hotmail.com

Universidade Federal do Espírito Santo - Centro de Ciências Jurídicas e Econômicas - Vitória - ES Brasil
\end{abstract}

\section{Teresa Cristina Janes Carneiro}

teresa.carneiro@gmail.com

Universidade Federal do Espírito Santo - Centro de Ciências Jurídicas e Econômicas - Vitória - ES Brasil

\section{Resumo}

As pesquisas que discutem a temática inovação e desempenho organizacional estão em evidência, pelo interesse das empresas, governos e instituições de pesquisa, fomentarem o desenvolvimento científico e tecnológico gerando resultados para a sociedade. Entretanto, pouco se sabe sobre a inovação e seu impacto sobre o desempenho organizacional. Dessa forma esse trabalho visa identificar os assuntos discutidos sobre essa temática, analisando as publicações da base Web of Knowledge que agrupa os principais periódicos internacionais sobre o tema. Esse trabalho identificou os assuntos que receberam mais atenção por parte dos pesquisadores e foi organizado em forma de uma pesquisa bibliométrica com base em 488 artigos selecionados. Com a utilização do software Alceste, dois eixos temáticos foram identificados, um com foco nos aspectos metodológicos dos indicadores de inovação e outro centrado nos aspectos humanos, sociais, econômicos e estratégicos da inovação e do desempenho organizacional.

Palavras-chave: Bibliometria, Alceste, Periódicos, Análise, Eixos Temáticos.

\section{Abstract}

The researches that discuss innovation and organizational performance are in evidence, as companies, governments and research institutions are interested in fostering scientific and technological development, thus generating results for society. However, little is known about innovation and its impact on organizational performance. Thus this paper aims to identify the subjects discussed on this topic, analyzing the publications in the Web of Knowledge database, which groups the main international journals related to innovation. This work identifies subjects that received the most attention from researchers and was organized as a bibliometric research based on 488 selected articles. Using the Alceste software, two thematic groups were identified, one focusing on methodological aspects of innovation indicators, and the other focusing on human, social, economic and strategic aspects of innovation and organizational performance.

Keywords: Bibliometrics, Alceste, Periodicals, Analysis, Thematic Groups. 


\section{Introdução}

Com rápido desenvolvimento da tecnologia e globalização dos mercados, a gestão organizacional tradicional deixa de ser a estratégia mais adequada para as organizações nesse ambiente em crescente mudança. Os negócios cada vez mais competitivos forcam as organizações a competir por sua sobrevivência através da melhoria contínua e inovação para sustentar vantagens competitivas. A inovação é vista como um importante instrumento nas estratégias de crescimento das organizações, para aumentar a participação de mercado, fornecer vantagem competitiva e melhorar o desempenho organizacional. Isso tem atraído atenção de muitos pesquisadores nas últimas décadas, que veem na inovação uma fonte de vantagem e uma alternativa de diferenciação frente aos demais concorrentes, além do potencial na melhoria de processos e do desempenho organizacional. Motivadas pelo aumento da concorrência nos mercados globais, às empresas começaram a compreender a influência e importância da inovação, uma vez que maiores graus de competição poderão resultar em taxas mais aceleradas de mudança tecnológica (UTTERBACK e SUAREZ, 1993). Assim, as inovações constituem um componente indispensável no desenvolvimento das estratégias corporativas, por atuarem nos processos de fabricação, melhorando o desempenho organizacional e a imagem transmitida aos clientes (JOHNE; Davies, 2000; Gonzalez-Benito, 2005).

O presente estudo utiliza o Manual de Oslo, (OCDE, 2005), principal base internacional de diretrizes para a definição e avaliação de atividades relacionadas à inovação, como fonte de referência básica para descrever, identificar e classificar a inovação no nível da organização. A inovação é caracterizada pelo Manual Oslo (OCDE, 2005) como a implementação de um produto (bem ou serviço) novo ou melhorado, processo novo, método de marketing novo ou método organizacional novo nas práticas de negócios, na organização do local de trabalho ou nas relações externas. Nesse contexto a inovação pode ser entendida como a utilização de uma invenção em algo prático, novo ou com melhorias, que associado às tecnologias existentes resulte em valor para o negócio.

No Manual Oslo da OCDE (2005), divide a inovação em quatro tipos: produto, processo, marketing e organização. A inovação de produto envolve características novas ou substancialmente melhoradas o serviço oferecido aos consumidores, já a inovação de processo envolve métodos, equipamentos e/ou habilidades para o desempenho do serviço novo ou substancialmente melhorado. A inovação de marketing introduz um novo método de marketing que gere mudanças significativas na concepção do produto e seu posicionamento de mercado. Inovação organizacional é caracterizada ela introdução de um novo método organizacional nas práticas empresariais que altere suas relações externas. Inovações organizacionais têm uma tendência de aumentar o desempenho da empresa, reduzindo custos administrativos e de transação, melhorando a satisfação no local de trabalho (GUNDAY, ULUSOY, KILIC e ALPKAN, 2011). Segundo Damanpour e Evan (1984), a inovação pode ser entendida como uma resposta da organização ao meio em que está inserida, favorecendo mudanças na estrutura e nos processos organizacionais.

As pesquisas tem despertado um interesse cada vez maior em entender como inovações geram vantagens competitivas às organizações. Portanto, o presente trabalho pretende analisar a abordagem da inovação relacionada ao desempenho organizacional por meio de uma pesquisa bibliográfica em uma base de dados que comporta os principais periódicos internacionais, a Web of Knowledge, coletando artigos disponíveis em todos os anos. Tal base de dados foi escolhida por representar uma das mais importantes bases de pesquisa, por possuir um vasto material disponível dos melhores periódicos internacionais. Procurou-se 


\section{INOVAÇÃO E DESEMPENHO ORGANIZACIONAL: UM ESTUDO DAS PUBLICAÇÕES CIENTÍFICAS DA BASE WEB OF KNOWLEDGE}

identificar a origem das principais contribuições sobre o tema, bem como os pesquisadores com maior número de publicações, os trabalhos mais citados e os periódicos onde o assunto tem maior destaque. Para que os objetivos fossem alcançados, recorreu-se a Bibliometria, uma técnica quantitativa e estatística usada para medir a produção e disseminação do conhecimento (ARAÚJO, 2006), para coletar os dados que foram analisados posteriormente, com o auxílio de software Alceste de análise textual.

0 trabalho foi organizado em 5 partes: a primeira é a introdução que inicia a discussão e situa o leitor sobre o assunto discutido, seguido pelo referencial teórico que aborda os tipos de inovação e as várias dimensões de desempenho, metodologia de pesquisa, resultados e análises da pesquisa e por fim as considerações finais.

\section{Inovação}

Diversos são os conceitos e teorias sobre a inovação. Soma-se a isso o fato do conceito que a maioria das pessoas tem difere dos conceitos acadêmicos, talvez por isso exista uma dificuldade de compreender e assimilar o significado da inovação em pesquisas científicas no ambiente organizacional. De acordo com o OCDE (2005, p. 55) “[...] a complexidade do processo de inovação e as variações na forma como ocorre nas empresas, faz com que seja necessário adotar convenções com o objetivo de fornecer definições operacionais que possam ser usadas em pesquisas padronizadas sobre empresas".

0 termo inovação tem conotação de algo novo, renovado. Nesse sentido inventar seria inovar, entretanto, Drucker (1986) distingue inovação de invenção, associando a primeira à ideia de explorar novas oportunidades e gerar valor à organização. Schumpeter (1934) descreveu os diferentes tipos de inovação: novos produtos, novos métodos de produção, novas fontes de fornecimento, a exploração de novos mercados e novas formas de organização empresarial. Nesse trabalho, a OCDE por meio do Manual de Oslo (OECD, 2005) - base primária internacional de diretrizes para definir e avaliar as atividades de inovação, bem como para a compilação e utilização de dados relacionados - foi tomado como fonte de referência fundamental para descrever, identificar e classificar inovações ao nível das empresas. 0 Manual de Oslo (OECD, 2005), faz uso de uma estrutura que abrange diversas visões de várias teorias da inovação baseadas na empresa e aborda a inovação como um sistema de inovação, que compreende:

- Inovação no produto - introdução de um bem ou serviço novo ou significativamente melhorado no que tange as suas características ou usos estimados;

- Inovação no processo - implementação de um método de produção ou distribuição novo ou significativamente melhorado. Incluem-se mudanças significativas em técnicas, equipamentos e/ou softwares;

- Inovação de marketing - implementação de um novo método de marketing com mudanças significativas na concepção do produto ou em sua embalagem, no posicionamento do produto, em sua promoção ou na fixação de preços;

- Inovação organizacional - implementação de um novo método organizacional nas práticas de negócios da empresa, na organização do seu local de trabalho ou em suas 
relações externas.

De acordo com a PINTEC (2008), inovação tecnológica é a introdução de um produto (bem ou serviço), processo produtivo tecnologicamente novo ou aprimorado; tendo sido desenvolvida pela empresa ou adquirida de outra empresa/instituição que a desenvolveu. A inovação é um dos instrumentos fundamentais de estratégias de crescimento para entrar em novos mercados, aumentar a quota de participação em mercado existente e fornecer à empresa vantagens competitivas (GUNDAY et al , 2011). Motivados pela concorrência acirrada nos mercados globais, às empresas começaram a entender a importância da inovação, uma vez que as mudanças tecnológicas rapidamente corroem o valor acrescentado dos produtos e serviços existentes.

Mercados mais dinâmicos forçam as organizações a mudar com maior velocidade, introduzindo novas tecnologias e repensando seus processos, a fim de adequar o negócio às novas exigências e necessidades dos clientes. Isso pode ser reforçado pelas pesquisas de Gopalakrishnan (2000) que identificou a velocidade da inovação assim como a magnitude da inovação são características relevantes, e que ambos tiveram um efeito positivo no desempenho da empresa. Freeman e Soete (2008) afirmam que as inovações são importantes não apenas para gerar riqueza, mas também para permitir que as pessoas façam coisas que não poderiam anteriormente. Isso tem relação com as inovações introduzidas na última década, que permitiram realizar transações através de aparelhos celulares, por exemplo, dinamizaram as relações de consumo.

\section{Desempenho Organizacional}

A avaliação do desempenho organizacional tem recebido crescente atenção dos pesquisadores nas últimas décadas, mas não há consenso quanto a sua operacionalização, já que embora a literatura sobre o tema disponibilize inúmeras medidas de desempenho, nenhuma, isoladamente, é capaz de abranger todos os aspectos relevantes do desempenho organizacional (COMBS; CROOK; SHOOK, 2005). Para Slack et al. (1997), a complexidade com que as empresas se apresentam no mercado impossibilita a redução do desempenho do negócio a um único indicador, exigindo assim a combinação de variados indicadores para que se amplie a análise do desempenho organizacional.

Os primeiros estudos sobre o desempenho, realizados nos anos 1950, procuraram identificar medidas que representassem as atividades dentro do contexto organizacional (MARTINDEL, 1950; RIDGWAY, 1956). Essa ideia foi reproduzida por Drucker (1954) ao desenvolver o que ficou conhecido como Administração por Objetivos. Os estudos de Drucker foram complementados por Koontz e O’Donnell (1974), mas o final da década de 1970 foi marcado por críticas aos modelos de mensuração das atividades estritamente internas à organização.

As pesquisas sobre mensuração do desempenho foram então direcionadas para o foco nos indicadores financeiros (retorno sobre ativos, retorno sobre patrimônio, retorno sobre as vendas). No entanto, a grande aceitação e utilização pelos pesquisadores de medidas financeiras na avaliação do desempenho não impediram que estas viessem também a se tornar alvo de críticas, porque tais modelos deixavam de lado indicadores de satisfação do consumidor, satisfação do funcionário, qualidade e inovação, considerados de grande importância para o desempenho do negócio (ITTNER; LARCKER, 1998). Às medidas de desempenho financeiro somaram-se então medidas de eficiência e eficácia das atividades do negócio ou desempenho operacional (NEELY, 2005) que compreende todas as medidas e indicadores estabelecidos para a avaliação das operações da organização (PERERA; HARRISON; POOLE, 1997). 


\section{INOVAÇÃO E DESEMPENHO ORGANIZACIONAL: UM ESTUDO DAS PUBLICAÇÕES CIENTÍFICAS DA BASE WEB OF KNOWLEDGE}

As informações de desempenho são utilizadas pelas organizações para direcionar seu planejamento, desenvolver estratégias que permitam aproveitar oportunidades, se defender de possíveis ameaças, além de auxiliar no entendimento dos resultados que sendo gerados pela transformação dos recursos materiais, humanos e tecnológicos. Neely et al (1995) salientam que o desempenho fornece ao gestor possibilidades de medir ações da organização, como sua capacidade produtiva, o nível de eficiência e eficácia dos processos, resultados das vendas, dessa forma existem diversos tipos de desempenho que são medidos de forma diferentes.

De acordo com a literatura, quatro diferentes dimensões de desempenho são utilizadas para representar o desempenho organizacional (Barringer e Bluedorn, 1999; Hagedoorn; Cloodt, 2003). Estas dimensões são desempenho inovativo, desempenho da produção, desempenho do mercado e desempenho financeiro.

Para medir o desempenho inovativo, os estudos em inovação e economia consideram o número de inovações patenteadas ou patenteáveis (novos processos, produtos ou tecnologias) como um fator importante, que fornece informações para calcular a criatividade e inovatividade de uma organização (Hagedoorn e Cloodt, 2003). 0 desempenho inovativo tem relação em maior e ou menor grau com as demais dimensões de desempenho, dependendo do ambiente em que a organização está inserida, a adoção de inovações em produtos, processos, marketing e inovações administrativas pode elevar o desempenho.

O desempenho da produção está relacionado com melhorias dos processos, eliminação do desperdício, otimização dos insumos utilizados na produção e melhoria do tempo de entrega. Pode ser entendido como a tendência da empresa em aplicar práticas de produção mais modernas e avançadas de gestão e de desempenho empresarial (Gonzalez-Benito, 2005). Segundo Gunday et al (2011) as melhorias em velocidade, qualidade, flexibilidade e eficiência de custos nas operações diárias conduziriam logicamente a melhoria da posição de mercado e maior retorno financeiro.

Satisfação do cliente, vendas totais, fatia de mercado são algumas medidas utilizadas para mensurar o desempenho do mercado, que está relacionado com a capacidade da organização de assumir uma posição de destaque dentro do seu segmento. Para Johne e Davies (2000) as inovações de marketing aumentam as vendas através elevação do consumo, que repercute em maior rendimento para as empresas. As inovações organizacionais são essenciais para o crescimento e o funcionamento eficaz de uma empresa, refletindo nas vendas, na fidelização dos clientes acesso a novos mercados, afetando positivamente o desempenho (Damanpour; Evan, 1984).

Frequentemente, medidas financeiras, como retorno sobre vendas (ROS), Retorno sobre Investimentos (ROI) e Retorno sobre Ativos (ROA) são utilizados para avaliação de desempenho financeiro (GUNDAY et al, 2011). Quando lidados com desempenho financeiro essas medidas talvez sejam as mais apropriadas, entretanto, outros tipos de desempenho necessitam de medidas próprias. De acordo com Eden e Hyndman (1999) no caso de organizações públicas, sem fins lucrativos, medir o desempenho está relacionado à eficiência dos serviços prestados, as razões principais para medir o desempenho é fornecer informações essenciais para melhorar a gestão e qualidade dos serviços, além de gerar dados para prestação de contas dos recursos utilizados. 
A partir de uma perspectiva sistêmica, o desempenho é a capacidade de uma organização em lidar com os quatro processos sistêmicos (entradas, saídas, transformações e efeitos de feedback) em relação ao alcance de objetivos ou metas (EVAN , 1976). A organização de alto desempenho deve realizar suas tarefas de forma eficiente, além de realizar sua função de adaptação de forma efetiva. A função de adaptação requer que a estrutura ou processos da organização passem por mudanças para atender às mudanças nas condições ambientais. Organizações inovadoras não apenas se adaptam à mudança ambiental, mas também utilizam seus recursos e habilidades para criar novas condições, como por exemplo, através da introdução de novos produtos ou serviços. Sendo assim, As inovações são meios de proporcionar mudanças internas ou externas, mantendo ou melhorando, dessa forma, o desempenho organizacional (DAMANPOUR; EVAN, 1984).

Grande parte das pesquisas sobre avaliação de desempenho concentra-se nas variáveis que podem ser medidas e na forma como a medição é operacionalizada, gerando informações numéricas de desempenho. Entretanto, o mais importante na medição não está nos valores gerados, muito menos nas medidas selecionadas, nem tampouco na forma como os dados são apresentados, mas nas decisões gerenciais e ações resultantes dessas medições que podem impactar a trajetória da organização e seus futuros resultados (Murphy; Trailer; Hill, 1996; Neely et al., 1995).

\section{Procedimentos metodológicos}

Este artigo consiste de uma pesquisa exploratória e bibliométrica. A Bibliometria foi desenvolvida segundo a lei de Zipf que "descreve a relação entre palavras num determinado texto suficientemente grande e a ordem de série destas palavras (contagem de palavras em largas amostragens)" (ARAÙJO, 2006, p.16), determinando a frequência e o peso que tem no texto.

Para entender a produção científica de pesquisas relacionadas com inovação e desempenho organizacional, foi realizada uma pesquisa exploratória e bibliográfica na base de dados Web of Knowledge de todos os anos disponíveis no banco de dados. A base de dados foi escolhida por representar uma das principais, contendo grande parte dos principais periódicos especializados em administração e economia classificados com o conceito "A" pelo Sistema Qualis da Capes (Coordenação de Aperfeiçoamento de Pessoal de Nível Superior). O conceito "A" tem relevância pelas características peculiares desses periódicos: originalidade, assertividade da pesquisa científica, adequação metodológica ao tema, relevância ou influência potencial, contribuições para o avanço do conhecimento científico e repercussão, popularidade ou visibilidade na comunidade acadêmica. Foram selecionados apenas artigos que estivessem disponíveis na base de dados Web of Knowledge na versão eletrônica. De acordo com o Qualis da Capes, na área de Administração, Ciências Contábeis e Turismo existem 158 periódicos classificados como "A1" e "A2". Na área de Economia existem 64 periódicos com a referida classificação. Alguns dos periódicos citados, em função das características das publicações, são avaliados em ambos as áreas do conhecimento.

Para selecionar os artigos na base de dados, foram utilizadas as palavras-chaves "innovation" e "performance", para buscar apenas artigos publicados na versão eletrônica que continham no título as palavras-chaves definidas. Os artigos não disponibilizados na base Web of Knowledge foram descartados, assim como os artigos não disponibilizados em formato digital. Utilizando esses critérios, foi feita uma busca, na qual resultou em 488 artigos completos publicados entre os anos de 1967 e 2012, dentre eles as revistas que mais se destacaram em número de publicações são categorizadas com o conceito "A" da Capes, demonstrando a importância da base pesquisada. 


\section{INOVAÇÃO E DESEMPENHO ORGANIZACIONAL: UM ESTUDO DAS PUBLICAÇÕES CIENTÍFICAS DA BASE WEB OF KNOWLEDGE}

Tabela 1

Número de periódicos por área e por classificação

\begin{tabular}{lrr}
\hline Área & A1 & A2 \\
\hline Administração, Ciências Contábeis e Turismo. & 76 & 82 \\
Economia & 28 & 36 \\
Total & 104 & 118 \\
Total das áreas & & $\mathbf{2 2 2}$ \\
\hline
\end{tabular}

Foram realizadas duas análises dos artigos selecionados na pesquisa. A primeira etapa foi feito um levantamento das publicações, separados por ano, por autor e nacionalidade. A segunda etapa foi verificada o conteúdo das palavras-chave, título e resumo, por meio do software de análise textual de dados Alceste. 0 Alceste classifica de maneira semi-automática as palavras para o interior de um corpus, estabelecendo as semelhanças entre os segmentos e hierarquias de classes de palavras, chamado de classificação método descendente hierárquico. O programa classifica as palavras conforme sua relevância no texto, que no caso foram os resumos de todos os artigos. Procurou-se identificar as temáticas centrais da discussão de inovação e desempenho, para isso utilizou-se a categorização em eixos temáticos e classes identificadas pelo Alceste. Os resultados são apresentados na sessão seguinte.

\section{Resultados e análises}

Buscando identificar a relação entre inovação e desempenho nas pesquisas publicadas em periódicos e disponíveis na base de dados Web of Knowledge, foi possível observar que a partir de 2007 a produção científica dessa temática ganhou maior importância no que tange volume de publicações, desenvolvendo uma trajetória ascendente, isso pode ser explicado em parte pela maior atenção dos pesquisadores no tema inovação.

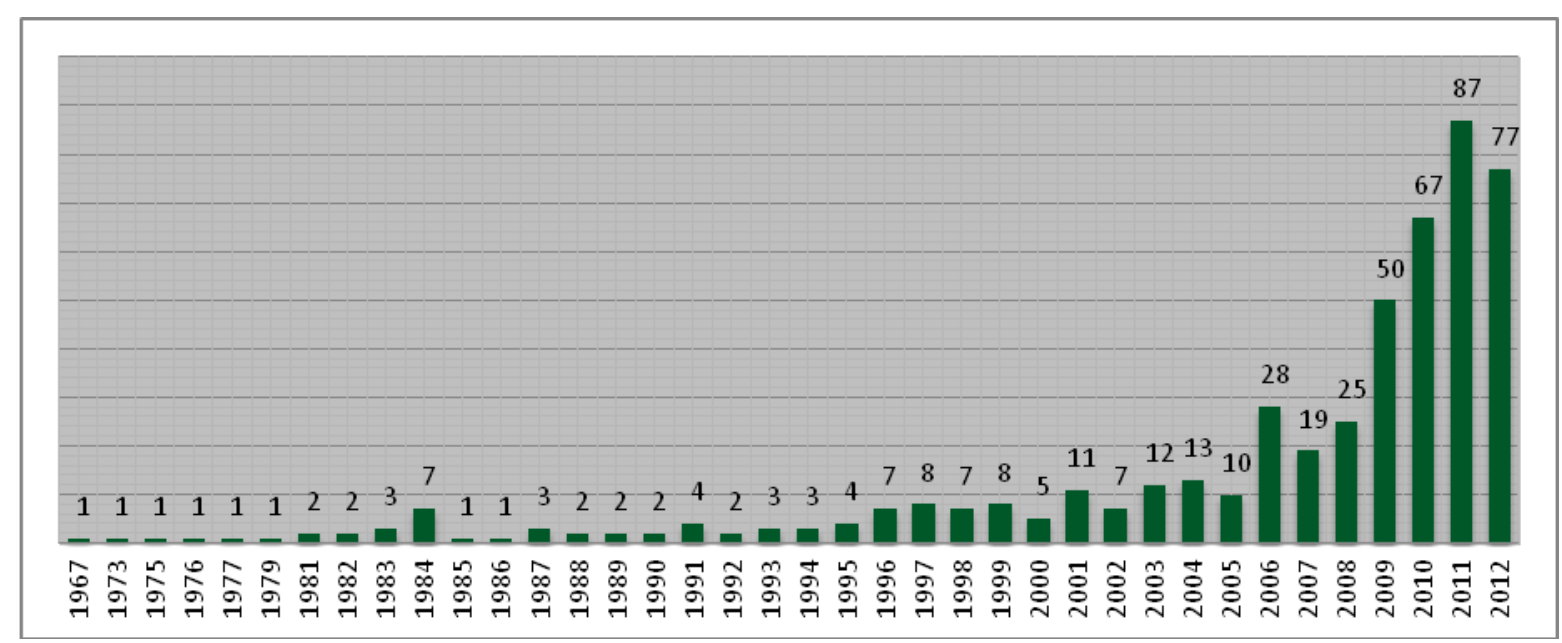

Gráfico 1 - Número absoluto de publicações científicas sobre o tema nos últimos 45 anos (1967-2012)

Analisando os resultados obtidos e compilados no gráfico 1, pode-se observar que as 
publicações obedeceram um padrão descontínuo durante a maior parte dos 45 anos analisados e que nos últimos 5 anos tem mostrado um tendência de aumento nas publicações da temática inovação e desempenho. Os aumentos mais acentuados ocorreram a partir de 2007, quando as publicações sobre inovação apresentaram uma tendência de crescimento que foi confirmada ano a ano. No ano de 2012 foram publicados 77 artigos sobre inovação, representando um acréscimo de $208 \%$ sobre as publicações do ano de 2008 , demonstrando como a temática tem ganhado relevância nos últimos 5 anos.

Tabela 2

\section{Publicação de artigos por periódico}

\begin{tabular}{lc}
\multicolumn{1}{c}{ Jornais } & $\begin{array}{c}\text { Qtde. de } \\
\text { srianc }\end{array}$ \\
\hline Technovation & 26 \\
International Journal of Technology Management & 18 \\
Journal of Product Innovation Management & 17 \\
Research Policy & 17 \\
African Journal of Business Management & 10 \\
Journal of Business Research & 10 \\
Strategic Management Journal & 10 \\
Industrial Marketing Management & 9 \\
International Journal of Production Economics & 7 \\
Industrial and Corporate Change & 6 \\
International Journal of Manpower & 6 \\
International Journal of operations Production Management & 6 \\
Small Business Economics & 6 \\
\hline
\end{tabular}

Dentre os periódicos com maior número de publicações, a revista Technovation destaca-se dentre as demais. A revista publicou um total de 26 artigos sobre a temática inovação e desempenho. Em segundo lugar, com 18 publicações no período, a revista International Journal of Technology Management, seguidas pela Journal of product Innovation management e o periódico Research Policy com 17 publicações cada uma.

Tabela 3

\section{Publicação de artigos por autor}

\begin{tabular}{lc}
\hline \multicolumn{1}{c}{ Autor } & Publicaçõe \\
\hline Victor J. Garcia-Morales & 5 \\
Kuen-Hung Tsai & 5 \\
Fariborz Damanpour & 4 \\
Dries Faems & 4 \\
Aron O'Cass & 4 \\
Zheng Michael Song & 4 \\
Richard C. Yam & 4 \\
Spyros Arvanitis & 3 \\
Kwaku AtuaheneGima & 3 \\
João Rodrigo Calantone & 3 \\
Cesar Camison & 3 \\
Yu-Shan Chen & 3 \\
\hline
\end{tabular}

A fim de entender as diferenças dos artigos publicados em diferentes periódicos, buscou-se entender as características dos artigos publicados em cada um dos periódicos. 0 periódico Technovation possui como foco publicações sobre as tendências tecnológicas, a disponibilidade de capital para o desenvolvimento de novos produtos, gestão de empreendimentos, gestão da inovação, estruturas organizacionais destinadas a facilitar a 


\section{INOVAÇÃO E DESEMPENHO ORGANIZACIONAL: UM ESTUDO DAS PUBLICAÇÕES CIENTÍFICAS DA BASE WEB OF KNOWLEDGE}

inovação, e de transferência de tecnologia para países em desenvolvimento. 0 segundo periódico com maior número de artigos publicados, International Journal of Technology Management, publica trabalhos sobre informação no domínio da gestão de tecnologia e gestão de ciência, engenharia e tecnologia. O Journal of Product Innovation Management, publica pesquisas relacionadas à teoria e prática no desenvolvimento de novos produtos e serviços. e o periódico Research Policy é uma revista multidisciplinar dedicada à análise de questões econômicas, políticas, de gestão, desafios organizacionais, ambientais e outros colocados pela inovação, tecnologia, R \& D e da ciência.

Dentre os pesquisadores com maior número de artigos científicos publicados sobre inovação e desempenho organizacional, destacam-se Kuen-Hung Tsai (professor do Departamento de Transportes da National Taiwan Ocean University) e Victor J. Garcia-Morales (professor da Faculdade de Ciências Econômicas e Empresarias da Universidade de Granada) com cinco publicações. Com quatro publicações o pesquisador Fariborz Damanpour (Professor de Administração na State University of New Jersey) destaca-se com o artigo "Organizational innovation and performance: the problem of organizational lag" pelo número de citações que o trabalho possui. Dries Faems (Professor de inovação e organização da Faculdade de Economia e Negócios da University of Groningen), Aron O'Cass ( Professor de Marketing na Escola de Gestão da Universidade da Tasmânia), Zheng Michael Song (professor no Departamento de Economia da Chinesa University of Hong Kong), e Richard C. Yam (professor da City University of Hong Kong ) destaca-se com 4 publicações.

Tabela 4

Países com maior número de autores afiliados

\begin{tabular}{lcc}
\hline \multicolumn{1}{c}{ País } & Qtde. de publicações & \% \\
\hline Estados Unidos & 131 & $28 \%$ \\
Taiwan & 62 & $13 \%$ \\
China & 42 & $9 \%$ \\
Inglaterra & 38 & $8 \%$ \\
Espanha & 38 & $8 \%$ \\
Holanda & 33 & $7 \%$ \\
Canadá & 30 & $6 \%$ \\
Alemanha & 22 & $4 \%$ \\
Austrália & 19 & $4 \%$ \\
Coréia do Sul & 17 & $3 \%$ \\
Itália & 13 & $2 \%$ \\
Bélgica & 9 & $2 \%$ \\
França & 9 & $2 \%$ \\
Suécia & 9 & $2 \%$ \\
Suiça & 8 & $1 \%$ \\
Brasil & 6 & $1 \%$ \\
Turquia & 6 & $1 \%$ \\
\hline
\end{tabular}

Entre os países com maior número de pesquisadores afiliados, o Brasil destaca-se com as publicações dos brasileiros, Maria Cléa Brito de Figueirêdo, Morsyleide de Freitas Rosa e Fernando Antônio Sousa de Aragão (pesquisadora da Embrapa Agroindústria Tropical); Geraldo Stachetti Rodrigues (pesquisador da Embrapa Labex Europe); Armando Caldeira- 
Pires (professor da Universidade de Brasília); Vicente de Paulo Pereira Barbosa Vieira e Francisco Suetônio Bastos Mota (professor da Universidade Federal do Ceará). Em conjunto publicaram dois artigos no Journal of Cleaner Production: "Environmental performance evaluation of agro-industrial innovations, part 1: Ambitec-Life Cycle, a methodo-logical approach for considering life cyclethinking" e "part 2: Methodological approach for performing vulnerability analysis of watersheds", no ano de 2010, contribuindo para o destaque do país em pesquisadores sobre inovação (Figueirêdo et al., 2010 e 2010). As informações sobre a universidades com maior número de pesquisadores filiados estão na tabela 4, demonstrando a posição de destaque das instituições chinesas em pesquisas sobre inovação.

Na segunda etapa da pesquisa, as palavras-chave, resumos, título dos artigos selecionados foram analisados com ajuda do software Alceste. 0 software trabalhou com as palavras-chave, títulos e resumos como unidades de contexto inicial (UCI), que são segmentos de texto composto do radical e suas variações. O conjunto das UCIs é definido como o corpus pelo software, com o qual foi gerado um documento a ser analisado. A Tabela 6 apresenta a análise das palavras-chave, mostrando a frequência que aparecem nas partes dos textos analisados.

Tabela 5

\section{Principais palavras-chaves e suas variações}

\begin{tabular}{lr}
\hline Palavra-chave & Qtde. \\
\hline Innovation+ & 204 \\
Perform+ & 165 \\
Firm+ + & 85 \\
Product+ & 43 \\
Relation+ & 37 \\
Effect+ & 36 \\
Technolog+ & 36 \\
Study+ & 36 \\
Market+ & 34 \\
Result+ & 30 \\
Research+ & 30 \\
Strateg+ & 30 \\
Organizational+ & 29 \\
Knowledge+ & 29 \\
Posit+ + & 27 \\
Industr+ & 27 \\
Develop+ & 25 \\
Model+ & 24 \\
New+ & 23 \\
Paper+ & 22 \\
Impac+ & 22 \\
Service+ & 20 \\
Show+ & 20 \\
Develop+ & 19 \\
Data+ & 18 \\
Manage+ & 18 \\
\hline & 18 \\
\hline
\end{tabular}




\section{INOVAÇÃO E DESEMPENHO ORGANIZACIONAL: UM ESTUDO DAS PUBLICAÇÕES CIENTÍFICAS DA BASE WEB OF KNOWLEDGE}

A palavra inovação e desempenho são as que mais citadas em função dos procedimentos de seleção dos artigos que as utilizaram como critério de busca. Pela frequência das palavras, um grupo está relacionado a questões metodológicas e ambientais (pesquisa, modelo, dados, método, desenvolvimento, efeito), um segundo grupo direciona para questões organizacionais (gestão, inovação, produto, empresa, organização, conhecimento) e um terceiro grupo de palavras refere-se às questões econômicas e estratégicas (estratégia, desempenho, marketing, novo, indústria, resultado, tecnologia).

A seguir é apresentado o Dendograma gerado pelo software Alceste com as nomeações dos eixos e classes, bem como as palavras que compõe cada núcleo. Os valores qui-quadrado $\left(\mathrm{X}^{2}\right)$ associados a cada palavra representam a importância semântica dentro da classe.

\begin{tabular}{|lc|}
\hline \multicolumn{2}{|c|}{ Classe 1 : (21 \%) } \\
\hline \multicolumn{2}{|c|}{ Indicadores de Inovação e } \\
Desempenho Organizacional \\
\hline \multicolumn{2}{|c|}{} \\
Palavras (UCE) & $\mathbf{X}^{\mathbf{2}}$ \\
\hline data+ & 98 \\
approach+ & 81 \\
technique+ & 56 \\
method+ & 50 \\
health+ & 48 \\
using+ & 47 \\
applic+ & 43 \\
statistical+ & 43 \\
analysis+ & 38 \\
concept+ & 37 \\
objetive+ & 36 \\
methodolog+ & 36 \\
weight+ & 36 \\
standard+ & 30 \\
compare+ & 30 \\
respect+ & 28 \\
system+ & 28 \\
\hline & 27 \\
\hline
\end{tabular}

\begin{tabular}{|c|c|c|c|}
\hline \multicolumn{2}{|c|}{ Classe 2: (51\%) } & \multicolumn{2}{|c|}{ Classe 3: (28\%) } \\
\hline \multicolumn{2}{|c|}{ Aspectos Humanos e } & \multicolumn{2}{|c|}{ Aspectos Econômicos e } \\
\hline \multicolumn{2}{|c|}{ Sociais } & \multicolumn{2}{|c|}{ Estratégicos } \\
\hline Palavras (UCE) & $\mathrm{X}^{2}$ & Palavras (UCE) & $\mathbf{X}^{2}$ \\
\hline organization+ & 94 & growth+ & 56 \\
\hline relation+ & 83 & export+ & 55 \\
\hline study+ & 67 & enterprise+ & 48 \\
\hline manage+ & 56 & firm+ & 44 \\
\hline implication+ & 56 & small+ & 41 \\
\hline research+ & 54 & trade+ & 36 \\
\hline perform+ & 45 & technology+ & 34 \\
\hline effect+ & 27 & r\&d & 33 \\
\hline project+ & 26 & econom+ & 33 \\
\hline understand+ & 26 & sale+ & 31 \\
\hline customer+ & 23 & high+ & 30 \\
\hline findings+ & 21 & presence+ & 30 \\
\hline antecedent+ & 21 & spillover+ & 30 \\
\hline innovation+ & 21 & product+ & 28 \\
\hline innovative+ & 21 & find + & 27 \\
\hline orientation+ & 20 & Local+ & 26 \\
\hline organ+ & 19 & emerg+ & 25 \\
\hline
\end{tabular}

Eixo 1: Indicadores de

Inovação e Desempenho

Organizacional

Eixo 2: Aspectos Humanos, Sociais, Econômicos e

Estratégicos da Inovação e Desempenho Organizacional

Figura 1 - Dendograma com classes e eixos estabelecidos pelo software Alceste

Foram analisados, a seguir, os títulos e resumos dos artigos selecionados. 0 corpus resultante 
dos 488 artigos apresentou 1.017 unidades de contexto elementar (UCEs) que representam um segmento de texto com sentido ou ideias frasais presentes no documento. 0 programa analisou $98 \%$ do material inicialmente processado, descartando apenas $2 \%$ que corresponde a títulos e resumos com pouca ou nenhuma relação entre si e com o restante do material analisado. A análise as UCEs permitiu obter classes por meio da classificação hierárquica descendente (CHD) que agrupa o vocabulário semelhante entre si, de acordo com a frequência e força da relação. A força da relação que une as classes é representada por um valor estatístico de qui-quadrado $\left(\mathrm{X}^{2}\right)$. Esse mecanismo permite classificar as relações obtidas pelas UCEs, categorizando em classes. A CHD dos títulos, resumos dos artigos selecionados identificou três classes que podem ser visualizadas no Dendograma figura 1.

A classe 1, com 214 UCEs representando 21\% do material analisado, foi denominada Indicadores de Inovação e Performance. Por não apresentar um índice de proximidade significativo com outras classes, constituirá um eixo temático com o mesmo nome da classe. A classe 2, que apresentou maior representatividade na pesquisa com 513 UCEs e $51 \%$ do material analisado, foi denominada Aspectos Operacionais da Inovação e Desempenho Organizacional. A classe 3 com 290 UCEs e 28\% do material pesquisado foi denominada Aspectos Econômicos e Estratégicos da Inovação e Desempenho Organizacional. A classe 2 e 3 apresentaram similaridades sendo agrupadas em um mesmo eixo temático denominado de Aspectos Operacionais, Econômicos e Estratégicos da Inovação e Desempeno Organizacional.

Os dois eixos temáticos obtidos através da análise de proximidades das palavras encontradas nos resumos e títulos dos artigos selecionados são apresentados a seguir.

\section{Eixo I: Indicadores de Inovação e Desempenho Organizacional}

0 primeiro eixo temático é constituído por uma única classe. Trata de assuntos relacionados à metodologia de medição e análise de dados através de abordagens estatísticas de indicadores de inovação e de desempenho.

\section{Classe 1: Indicadores de Inovação e Desempenho Organizacional}

Nessa classe foram classificados os artigos que discorrem sobre indicadores de inovação e desempenho em organizações de várias áreas: saúde, educação, agronegócio, meio ambiente. Há artigos que propõem novas metodologias de medição tais como lógica Fuzzi e Análise Envoltória de Dados (DEA). No artigo "Regional innovation systems: how to assess performance", Zabala-Iturriagagoitia et al (2007) aplicam a análise envoltória de dados na avaliação do desempenho do sistema regional de inovação com base em informações fornecidas pelo European Innovation Scoreboard (EIS). No artigo "The measurement of technical performance of innovations by technometrics and its impact on established technology indicators", Grupp (1994) propõe um novo indicador para analisar os retornos de pesquisa, desenvolvimento e inovação (P\&D), em substituição a medidas diretas de especificação de tecnologia (Technometrics) que utilizam análise de patentes. Em "Application of neuro_fuzzy networks to forecast innovation performance: the example of taiwanese manufacturing industry", Chiena, Wangb e Lina (2010) elaboram um modelo de rede neural para prever o desempenho da inovação com regras fuzzy e medir o desempenho da inovação.

0 modelo auxilia os tomadores de decisão na alocação eficiente de recursos visando atingir os objetivos da inovação. Outros dois artigos que tem forte relação com essa classe são "Meditation, learning, organizational innovation and performance", em que Ho (2010) aborda a importância das medições de estresses e as implicações gerencias que impactam no desempenho organizacional e "A method for evaluating the overall technical and economic performance of environmental innovations in productions cycles", em que Barbirolli e Raggi 


\section{INOVAÇÃO E DESEMPENHO ORGANIZACIONAL: UM ESTUDO DAS PUBLICAÇÕES CIENTÍFICAS DA BASE WEB OF KNOWLEDGE}

(2003) propõem um método quantitativo de classificação das inovações de produção mais limpas com base em critérios técnicos e econômicos, abordando assuntos relacionados à ecoeficiencia.

Nessa classe estão reunidos artigos publicados prioritariamente em periódicos da área de gestão da tecnologia (Ex: Journal of Cleaner Production, IEEE Transactions on Information Theory, Exper Systems with Applications).

\section{Eixo II: Aspectos Humanos, Sociais, Econômicos e Estratégicos da Inovação e Desempenho Organizacional}

Esse eixo temático reúne a parte mais significativa dos artigos publicados sobre inovação e desempenho, representando $79 \%$ do material analisado. Nele são discutidos os aspectos humanos e sociais envolvidos no processo de inovação e mudanças relacionadas à inovação e os benefícios econômicos e financeiros da inovação. Em "How top management team diversity affects innovativeness and performance via the strategic choice to focus on innovation fields", Talke, Salomo e Rost (2007) pesquisam sobre as equipes de alta gestão, o tipo de gerência relacionada à estratégia adotada pela organização, que pode repercutir em melhor desempenho e facilitar uma estratégia de inovação de produtos.

Outro artigo com forte correlação com o eixo temático, "Innovation and export performance: evidence from the UK and German manufacturing plants", Roper e Love (2002) encontraram evidências positivas da relação entre a capacidade inovativa e a propensão em exportar, em pesquisa realizada em plantas industriais de empresas alemãs. Analisando as questões econômicas, Kannebley, Sekkel e Araújo (2010), no trabalho "Economic performance of Brazilian manufacturing firms: a counterfactual analysis of innovation impacts", correlacionaram os efeitos da inovação tecnológica com impactos positivos sobre o emprego, a receita líquida, a produtividade do trabalho e participação de mercado, demonstrando que a inovação pode ser um caminho para obtenção de vantagens competitivas. Esse eixo é subdivido em duas classes apresentadas a seguir.

\section{Classe 2: Aspectos Humanos e Sociais da inovação e desempenho organizacional}

Nessa classe estão reunidos pesquisas que abordam os temas orientação para o mercado e para os clientes e aspectos humanos relacionados à inovação: gestão do conhecimento, gestão de conflitos e da mudança, atitude gerencial, liderança, motivação, relacionamento entre equipe. Em "Innovation is not enough: climates for initiative and psychological safety, process innovations, and firm performance", Baer e Frese (2003) estudam a relação entre o clima organizacional e a segurança psicológica na adoção e implementação de inovações de processos e seu efeito benéfico no desempenho, um estudo realizado em empresas de diversos segmentos na Alemanha.

Artigos com forte correlação com o eixo temático são: (a) "Tangible slack versus intangible resources the influence of technology slack and tacit knowledge on the capability of organisational learning to generate innovation and performance" em que Bueno et al. (2010) identificam a influência forte que recursos intangíveis representados pelo conhecimento tácito exercem sobre a aprendizagem organizacional, inovação e desempenho em comparação com o impacto mais moderado dos recursos tangíveis relacionados à folga tecnológica; (b) 
"Strategic human resource practices and innovation performance: the mediating role of knowledge management capacity" em que Chen e Huang (2009) mostram que as práticas estratégicas de recursos humanos estão positivamente relacionadas com a capacidade de gestão do conhecimento que, por sua vez, tem um efeito positivo sobre o desempenho da inovação; (c) "Improving employees' job satisfaction and innovation performance using conflict management" em que Chen et al. (2012) identificam que a gestão de conflitos está positivamente relacionada com a satisfação no trabalho que por sua vez está positivamente relacionada com o desempenho da inovação e que evitar comportamentos de gestão de conflitos impacta negativamente o desempenho da inovação; (d) "The moderating role of organizational context on the relationship between innovation and firm performance" em que De Clercq et al. (2011) analisam a forma como a autonomia de decisão, a confiança e o comprometimento organizacional, tanto individual como coletivo, agem como catalisadores da capacidade da empresa em converter suas atividades de inovação em resultados de desempenho.

Os artigos agrupados nessa classe são prioritariamente de periódicos da área de Administração (Ex: International Journal of Research in Marketing, Leadership Quarterly, Journal of Business Research, International Journal of Conflict Management, Journal of Marketing, Journal of Organizational Change Management).

Classe 3: Aspectos Econômicos e Estratégicos da inovação e desempenho organizacional

Nessa classe são discutidos os benefícios econômicos da inovação, utilizando métricas predominantemente financeiras para medir o desempenho. Em "The impact of innovation on economic performance in services", Cainelli, Evangelista e Savona (2004) estudam os impactos da inovação no desempenho financeiro da organização em termos de produtividade e de crescimento, investigando empresas prestadoras de serviços. Identificam que altos níveis de produtividade e inovação têm relação com crescimento organizacional. Nessa classe estão as questões estratégicas da inovação, como o efeito das alianças entre empresas para desenvolvimento de novos produtos. Em "Product innovation strategy and performance of new technology ventures in China", Li e Atuahene-gina (2001) investigam os efeitos da inovação de produto sobre o desempenho em empresas de base tecnológica na China, sob a ótica da adoção dessa estratégia e as vantagens competitivas resultantes. As estratégias de colaboração e proteção da inovação são tratadas por Teece (1986) em "Profiting from technological innovation: implications for integration, collaboration, licensing and public policy", demonstrando que inovar não é suficiente para manter uma posição destaque no mercado e que estratégicas que considerem a integração e colaboração podem ser importantes para gerar vantagens competitivas sustentáveis.

Os artigos agrupados nessa classe são, em sua maioria, publicações de periódicos da área de Economia e Estratégia (Ex: Australian Economic Review, European Planning Studies, Industrial and Corporate Change, Service Industries Journal, Small Business Economics, Strategic Management Journal, Technovation).

\section{Considerações finais}

Esse trabalho se propôs realizar uma análise bibliométrica sobre os temas inovação e desempenho organizacional, em artigos disponíveis na base de dados Web of Knowledge. Utilizando-se como critério de busca as palavras "inovação" e "desempenho" no tópico, foi possível coletar informações dos últimos 45 anos de publicações, observando o avanço conjunto dos dois temas ao longo dos anos. Constatou-se que a temática vem ganhando atenção dos pesquisadores ao longo desse período de tempo, em particular nos últimos cinco 


\section{INOVAÇÃO E DESEMPENHO ORGANIZACIONAL: UM ESTUDO DAS PUBLICAÇÕES CIENTÍFICAS DA BASE WEB OF KNOWLEDGE}

anos, mostrando um aumento do interesse do tema em pesquisas realizadas nos campos de estudos organizacionais, estratégia e marketing.

O periódico Technovation apresentou o maior número de artigos sobre o tema. Possui como foco as tendências tecnológicas, gestão da inovação, transferência de tecnologia para países em desenvolvimento, justificando sua posição de destaque em publicações dessa temática. 0 segundo periódico com maior número de artigos publicados, International Journal of Technology Management, publica trabalhos sobre gestão da tecnologia, representando um campo propício para tratar de inovação. Os autores com maior número de publicações foram Kuen-Hung Tsai (Professor do Departamento de Transportes da National Taiwan Ocean University), Fariborz Damanpour (Professor de Administração na State University of New Jersey) e Dries Faems (Professor de Inovação e Organização da Faculdade de Economia e Negócios da University of Groningen), indicando que o tema é multidisciplinar de interesse de centros de pesquisa localizados na Europa, América e Ásia.

A análise dos títulos e resumos dos artigos selecionados com auxílio do software de análise textual Alceste, permitiu a identificação de dois grupos distintos de assuntos abordados: um grupo mais metodológico, voltado para a discussão de métricas e indicadores de inovação e seus resultados e outro focado nos aspectos humanos, sociais e econômicos da inovação e do desempenho organizacional. Esse último grupo, foi subdividido em duas classes distintas. A primeira classe denominada "aspectos humanos e sociais da inovação e desempenho organizacional" reuniu pesquisas que tratam de temas da área de administração, envolvendo aspectos das mudanças organizacionais envolvidas na adoção de inovações e seus resultados. Dentre os assuntos abordados podem-se citar aspectos humanos relacionados à gestão do conhecimento, gestão de conflitos e da mudança, atitudes gerenciais e estilos de liderança que propiciam a inovação, motivação para inovar, relacionamento entre equipes envolvidas nos processos de lançamento ou adoção de inovações em produtos e processos. A segunda classe desse grupo, denominada "aspectos econômicos e estratégicos da inovação e desempenho organizacional" reuniu pesquisas que abordavam os benefícios da inovação para setores econômicos ou para a sociedade, utilizando métricas predominantemente financeiras para o desempenho.

A análise de conteúdo dos títulos e resumos dos artigos selecionados permitiu identificar que os aspectos envolvendo inovação e desempenho organizacional são de interesse de várias áreas do conhecimento, com abordagens teóricas e metodológicas distintas e níveis da análise que variam desde abordagens no nível micro da gestão de recursos humanos, passando pelo plano meso das organizações até o nível macro dos setores produtivos e das políticas e sistemas de inovação. 0 desempenho organizacional obtido por estratégias tanto de adoção como de lançamento de inovações é avaliado tanto internamente às organizações como externamente nos setores produtivos.

Em função da quantidade de dados levantados pela pesquisa, alguns tópicos merecem aprofundamento em pesquisas futuras. 0 presente trabalho contribui para melhor compreensão dos estudos em inovação que se relacionam com o desempenho organizacional ao trazer um mapeamento das principais publicações. Dessa forma os dados analisados mostram que o tema é interdisciplinar permitindo abordagens sob várias perspectivas teóricas e metodológicas. 


\section{Referências}

Amaratunga, D.; BALDRY, D. Performance evaluation in facilities management. In: Proceedings of COBRA, Greenwich, UK, 2000.

Araújo, C.A. Bibliometria: evolução histórica e questões atuais. Em Questão, v. 12, n. 1, p. 1132, 2006.

BAER, M.; FRESE, M. Innovation is not enough: climates for initiative and psychological safety, process innovations, and firm performance. Journal of Organizational Behavior. v.24, p. 4568, 2003

BARBIROLLI, G.; RAGGI, G. A method for evaluating the overall technical and economic performance of environmental innovations in productions cycles. Journal Cleaner Production, v.11, n.4, p. 365-374, 2003.

Barringer, B.R.; Bluedorn, A.C. The relationship between corporate entrepreneurship and strategic management. Strategic Management Journal, v.20, n.5, p.421-444, 1999.

Bassioni, H. A.; PRICE, A. D. F.; Hassan, T. M. Performance measurement in construction firms. Journal of Management in Engineering, v. 20, n.2, p.42-50, 2004.

BUENO, E.; ARAGON, J. A.; SALMADOR, M. P.; GARCIA, V. J. Tangible slack versus intangible resources: the influence of technology slack and tacit knowledge on the capability of organisational learning to generate innovation and performance. International Jounal of Technology Management, vol.49, n.4, p.314-337, 2010.

Cainelli, G.; Evangelista, R; SAVONA, M. The impact of innovation on economic performance in service. Service Industry Journal, v.24, n. 1, p. 1-11, 2004.

Carrión-Flores, C.; Innes, R. Environmental innovation and environmental performance. Journal of Environmental Economics and Management, v.59, n.1, p.27-42, 2010.

CHEN, X.; ZHAO, K., LIU, X, WU, D. D. Improving employees' job satisfaction and innovation performance using conflict management. International Journal of Conflict Management, vol. 23, n. 2, p.151 - 172, 2012.

CHEN, C.; HUANG, J. Strategic human resource practices and innovation performance: the mediating role of knowledge management capacity. Journal of Business Research, vol. 62, $\mathrm{n}$. 1, p.104-114, 2009.

CHIEN, S.; WANG, T.; LIN, S. Application of neuro-fuzzy networks to forecast innovation performance: the example of Taiwanese manufacturing industry. Expert Systems with Applications, vol. 37, n.2, p.1086-1095, 2010.

COMBS, J. G.; CROOK, T. R.; SHOOK, C. The dimensionality of organizational performance and its implications for strategic management research. In: Research Methodology in Strategy and Management, San Diego, CA: Elsevier. p. 259-286, 2005.

DAMANPOUR F., EVAN; W.M. Organizational innovation and performance: the problem of "organizational lag". Administrative Science Quarterly, v. 29, p.392-409, 1984. 


\section{INOVAÇÃO E DESEMPENHO ORGANIZACIONAL: UM ESTUDO DAS PUBLICAÇÕES CIENTÍFICAS DA BASE WEB OF KNOWLEDGE}

Damanpour, F. The adoption of technological, administrative, and ancillary innovations: impact of organizational factors. Journal of Management, v.13, n.4, p. 675-688, 1987.

DE CLERCQ, D.; THONGPAPANL, N.; DIMOV, D. The Moderating role of organizational context on the relationship between innovation and firm performance. IEEE Transactions on Engineering Management, 58(3), 431-444, 2011.

DRUCKER, P.F. Inovação e espírito empreendedor (entrepreneurship): prática e princípios. São Paulo: Pioneira, 1986.

The pratice of management. New York: Harper, 1954.

Eden, R.; HYNDMAN, N. Performance measurement in the UK public sector: poisoned chalice or Holy Grail? Optimum: The Journal of Public Sector Management, v. 29, n. 1, p.9-15, 1999.

EVAN, W. M. Organization theory and organizational effectiveness: an exploratory analysis. Organization and Administrative Sciences, v.7, p.16-28, 1976.

Evangelidizs, K. Performance measured is performance gained. The Treasurer, p.45-47, 1992.

FIGUEIREDO, M. C. B.; RODRIGUES, G. S.; CALDEIRA-PIRES, A.; ROSA, M. F.; ARAGÃO, F. A. S.; VIEIRA, V. P. P. B.; MOTA, F. S. B. Environmental performance evaluation of agro-industrial innovations - part 1:Ambitec-life cycle, a methodological approach for considering life cycle thinking. Journal of Cleaner Production, v. 18, n. 14, p. 1366-1375, 2010.

Environmental performance evaluation of agro-industrial innovations - part 2: methodological approach for performing vulnerability analysis of watersheds. Journal of Cleaner Production, v. 18, n. 14, p. 1376-1385, 2010.

FREEMAN, C.; SOETE, L. A economia da inovação industrial. Campinas: Editora da Unicamp, 2008.

Gatignon, H.; Tushman, M.L.; Smith, W.; Anderson, P. A structural approach to assessing innovation: construct development of innovation locus, type, and characteristics. Management Science, v.48, n.9, p.1103-1122, 2002.

Gonzalez-Benito, J. A study of the effect of manufacturing proactivity on business performance. International Journal of Operations and Production Management. v.25, n.3-4, p.222-241, 2005.

Gopalakrishnan, S. Unraveling the links between dimensions of innovation and organizational performance. Journal of High Technology Management Research, v.11, n.1, p.137-153, 2001.

GRUPP, H. The measurement of technical performance of innovations by technometrics and its impact on established technology indicators. Research Policy, vol. 23, n. 2, p.175-193, 1994. 
GUNDAY, G.; ULUSOY. G.; KILIC, K.; ALPKAN, L. Effects of innovation types on firm performance. International Journal Production Economics, v. 133, n.2, 2011.

Hagedoorn, J.; Cloodt, M. Measuring innovative performance: is there an advantage in using multiple indicators? Research Policy v. 32, n.8, p.1365-1379, 2003.

Ho, L. Meditation, learning, organizational innovation and performance. Industrial Management \& Data Systems, v.111, n.1, p. 113-131, 2011.

IBGE. Pesquisa de Inovação Tecnológica (PINTEC). 2008. Disponível em <http://www.pintec.ibge.gov.br>. Acesso em 20 de julho de 2012.

ITTNER, C.D.; LARCKER, D.F. Are nonfinancial measures leading indicators of financial performance? An analysis of customer satisfaction. Journal of Accounting Research, v. 36, p. $1-35,1998$.

Johne, A.; Davies, R. Innovation in medium-sized insurance companies: how marketing adds value. International Journal of Bank Marketing. v. 18, n.1, p. 6-14, 2000.

Kagioglu, M.; Cooper, R.; Aouad, G. Performance management in construction: a conceptual framework. Construction Management and Economics, v. 19, n.1, p. 85-95, 2001.

Kannebley, S.; SEKKEL J. V.; Araújo, B.C. Economic performance of brazilian manufacturing firms: a counterfactual analysis of innovation impacts. Small Business Economics, v.34, n.3, p.339-353, 2010.

KOONTZ, H.; O’DONNELL, C. Princípios de administração. São Paulo: Pioneira Thomson Learning, 1974.

LI, H.; ATUAHENE-GIMA, K. Product innovation strategy and performance of new technology ventures in China. Academy of Management Journal, v 44, n.6, p. 1123-1134, 2001.

MARTINDEL, J. The scientific appraisal of management: a study of the business practices of the well managed companies. New York: Harper, 1950.

Murphy G.B.; Trailer, J.W.; Hill, J.W. Measuring performance in entrepreneurship research. Journal of Business Research, v. 36, n.1, p.15-23, 1996.

NEELY, A. The evolution of performance measurement research: developments in the last decade and a research agenda for the next. International Journal of Operations \& Production Management, v. 25, n.12, p. 1264 -1277, 2005.

Neely, A.D; Gregory, M.J.; Platts, K.W. Performance measurement system design: a literature review and research agenda. International Journal of Operations \& Production Management, v. 15, n. 4, p. 80-116, 1995.

OECD. Manual de Oslo: Diretrizes para coleta e interpretação de dados sobre inovação. $3^{a}$. ed. OECD e Eurostat. 2005.

PERERA, S.; HARRISON, G.; POOLE, M. Customer-focused manufacturing strategy and the use of operations-based non-financial performance measures: a research note. Accounting, Organizations and Society, v.22, n.6, p.557-572, 1997. 


\section{INOVAÇÃO E DESEMPENHO ORGANIZACIONAL: UM ESTUDO DAS PUBLICAÇÕES CIENTÍFICAS DA BASE WEB OF KNOWLEDGE}

RIDGWAY, V. F. Dysfunctional consequences of performance measurements. Administrative Science Quarterly, v.1, n.2, p. 240 - 247, 1956.

ROPER, S.; LOVE, J.H. Innovation and export performance: evidence from the UK and German manufacturing plants. Research Policy, v.31, n, 7, p. 1087-1102, 2002.

SAQUETO, T.C.; CARNEIRO, T.C.J. Inovação tecnológica: análise da publicação científica de 2001 a 2010. Análise, v.22, n1, p.17-30, 2011.

Schumpeter, J.A. The theory of economic development an inquiry into profits, capital, credit, interest, and the business cycle. Harvard University Press, Cambridge, 1934.

SLACK, N; et al. Administração da produção. São Paulo: Atlas. 1997.

Talke, K.; Salomo, S.; ROST, K. How top management team diversity affects innovativeness and performance via the strategic choice to focus on innovation fields. Research Policy, v.39, n.7, p. 907-918, 2007.

TEECE, D.J. Profiting from technological innovation: implications for integration, collaboration, licensing and public policy. Research Policy, v. 15, p. 285-305, 1986.

UTTERBACK, J.M.; SUAREZ, F.F. Innovation, competition, and industry structure. Research Policy, v. 22, p. 1-21, 1993.

YAM, R.C.M.; LO, W.; TANG, E.P.Y.; LAU, A.K.W. Analysis of sources of innovation, technological innovation capabilities, and performance: an empirical study of Hong Kong manufacturing industries. Research Policy, v.40, n.3, p. 391-402, 2011.

ZABALA-ITURRIAGAGOITIA, J. M.; VOIGT P.; GUTIÉRREZ-GRACIA, A.; JIMÉNEZ-SÁEZ F. Regional innovation systems: how to assess performance, Regional Studies, v.41, p.661-672, 2007. 